\title{
An 8-20-GHz Wide-Band LNA Design and the Analysis of Its Input Matching Mechanism
}

\author{
Robert $\mathrm{Hu}$
}

\begin{abstract}
In this letter, a wide-band low-noise amplifier (LNA) design is presented with $-10 \mathrm{~dB}$ input reflection coefficient and 100-K noise temperature over the $8-20-\mathbf{G H z}$ frequency range at room temperature. This LNA can be cooled down in a liquid Helium cryostat to have 10-K noise temperature for the purpose of radio-astronomy application. To achieve its wide-band characteristics, a novel input matching mechanism is proposed, which combines the high-frequency inductive feedback and the low-frequency capacitive feedback. The recognition and comprehensive interpretation of this new input matching mechanism is crucial for future development of ultra-wide-band LNAs.
\end{abstract}

Index Terms-Input matching, low-noise amplifier (LNA), wideband.

\section{INTRODUCTION}

$\mathbf{T}$ HE WIDE-BAND low-noise amplifier (LNA) is in high demand in the radio-astronomy community [1], [2]. While the simplest way of achieving a small input reflection coefficient $\left(S_{11}\right)$ over a wide bandwidth is to add either a shunt resistor or a feedback resistor to the circuit's input-stage transistor, the drawbacks, however, are the attenuation of the incoming signal and the additional thermal noise which, thus, result in a noisy amplifier. On the other hand, when a source inductor and an input series inductor are connected to this transistor, it can only have a small $S_{11}$ and low noise temperature $\left(T_{n}\right)$ over a relatively narrow bandwidth [3]. Therefore, wide-band LNAs used in radio-astronomy receivers are conventionally implemented either by using the balanced circuit configuration, which has twice the power dissipation [4], or by adding a bulky isolator in front of the amplifier to loosen the $S_{11}$ constraint [5], [6].

In this letter, an 8-20-GHz wide-band LNA is designed by resorting to the transistor's intrinsic feedback mechanism, i.e., via the gate-drain capacitor $C_{\mathrm{gd}}$. Through deliberately fine-tuning the first-stage transistor's loading impedance, the resulting $S_{11}$ can be small over a broad bandwidth. Section II will present the simulated and measured results.

Though circuit simulation alone can show that a transistor with an external source inductor and a $R C$ loading impedance could provide a wide-band input match and a flat noise temperature, the challenge, however, is on constructing the mathematical formulation and a comprehensive interpretation. In Section III, two narrow-band lossless feedback mechanisms are analyzed where the inductive feedback is interpreted as a high-fre-

Manuscript received March 4, 2004; revised June 4, 2004. This work was supported in part by NASA under Grant NAG5-9493. The review of this letter was arranged by Associate Editor A. Stelzer.

The author is with the Department of Electronics Engineering, National Chiao Tung University, Hsinchu, Taiwan, R.O.C.

Digital Object Identifier 10.1109/LMWC.2004.837063

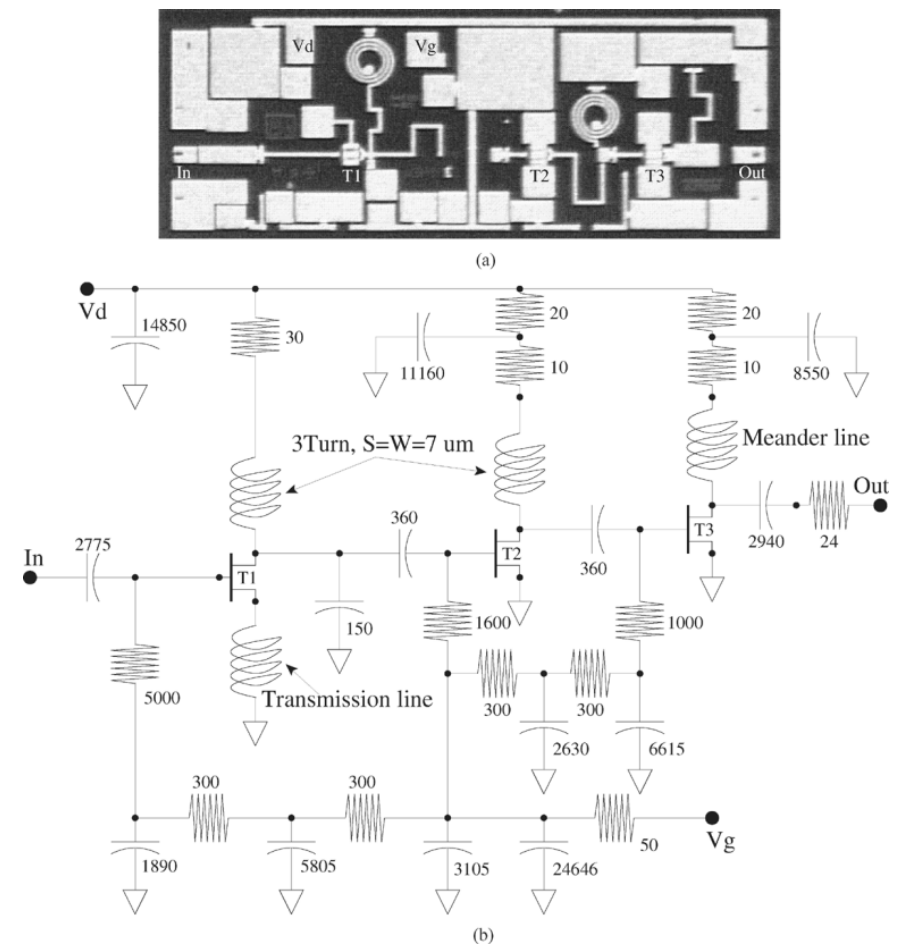

Fig. 1. The $8-20-\mathrm{GHz}$ wide-band LNA. (a) This wide-band circuit is fabricated by TRW and has dimensions of $2000 \times 750 \times 75 \mu \mathrm{m}^{3}$. It is a three-stage circuit with transistors T1, T2, and T3. (b) Schematic. The resistance is in ohms $(\Omega)$, capacitance in $\mathrm{fF}$ and inductance in $\mathrm{pH}$. The two spiral inductors on the drain branches are each three turns with $7-\mu \mathrm{m}$ line separation $(S)$ and $7-\mu \mathrm{m}$ linewidth $(W)$.

quency input matching mechanism while the capacitive feedback is treated as low-frequency input matching. The new type of wide-band feedback can therefore be easily understood by combining these two well-explained narrow-band mechanisms.

\section{8-20-GHz WIDE-BAND LNA DESIGN}

An 8-20-GHz LNA was designed using the Agilent's advanced design system (ADS). This LNA is a three-stage circuit with all three transistors operating at the same bias condition (Fig. 1). Specifications of the passive components are $100 \Omega$ /square for thin-film resistors, $0.3 \mathrm{fF} / \mu \mathrm{m}^{2}$ for thin-film capacitors, and $100 \times 100 \mu \mathrm{m}$ reserved space for via pad.

In this circuit, the transistor's gate-bias circuits are each made of a high-value resistor, instead of a quarter-wave stub that is narrow-band and hard to implement in this frequency range. To further minimize its noise impact, the width of the first gate-bias resistor is narrowed down to $2 \mu \mathrm{m}$. The spiral inductors on the first two transistors' drain-bias branches have large impedance at this frequency range; therefore, they can boost the gain of the 


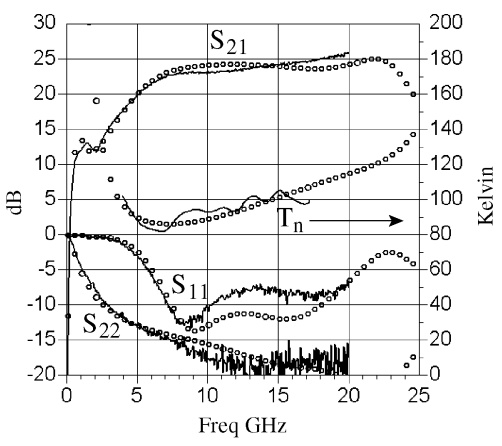

(a)

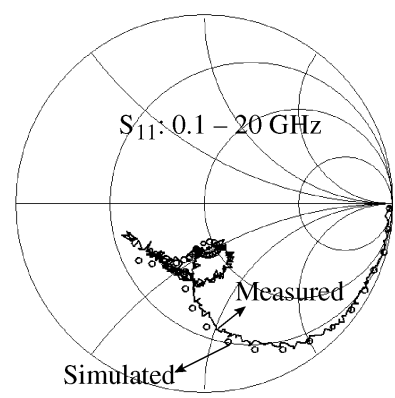

(b)

Fig. 2. Simulated and measured results of the wide-band LNA on-wafer at room temperature. (a) The circled curves are the simulated results where the drain temperature for the noise temperature calculation is set to $2500 \mathrm{~K}$. The solid curves are the measured results where the frequency range is limited by the network analyzer and the noise source available at the time of the measurements. The dc bias is $V_{d}=0.8 \mathrm{~V}$ and $I_{d}=36 \mathrm{~mA}$, so the total power dissipation is $28.8 \mathrm{~mW}$. (b) $S_{11}$ on the Smith chart. Later in the packaging phase, a $200-300-\mu \mathrm{m}$ gold bond-wire at this circuit's input will move the contour closer to the origin point and bring down $S_{11}$ further, as has been considered in the circuit design.

transistor and thus reduce the noise contribution from the subsequent stages. The use of the meander line instead of a spiral inductor on the last transistor's drain-bias branch can lower the circuit's output inductance. These bias circuits, together with the interstage dc blocking capacitors, also provide the necessary gain tapering. The small-signal model of the transistor used in the simulation is obtained analytically [7], [8]. The transistor's noise behavior is modeled by assigning a temperature $T_{d}$ to the intrinsic drain resistor $R_{\mathrm{ds}}$ while retaining all the other components at ambient temperature [9]. The simulated and measured results are shown in Figs. 2 and 3.

\section{ANALYSIS OF INPUT-MATChING MECHANISM}

In the inductive feedback, as in Fig. 4(a), but with the loading $R C$ circuit replaced by $Z_{0}(50 \Omega)$, the induced current on the drain side flowing through the source inductor $L_{F B}$ will generate a voltage that is in phase with the input current. Inductor $L_{F B}$ can therefore be treated, from the input perspective, as a resistor. Mathematically, the input impedance $Z_{\text {in }}$ of the circuit is a capacitor in series with a resistor

$$
Z_{\text {in }}=\left(\frac{1}{j \omega C_{\mathrm{gs}}}+\frac{L_{F B} \gamma G_{m}}{C_{\mathrm{gs}}}\right)\left[1+\frac{C_{\mathrm{gd}}}{C_{\mathrm{gs}}}\left(1+\gamma G_{m} Z_{0}\right)\right]^{-1}
$$

where $\gamma=R_{\mathrm{ds}} /\left(R_{\mathrm{ds}}+Z_{0}+j \omega L_{F B}\right)$ is the degradation factor to the transconductance $G_{m}$. On the Smith chart, the

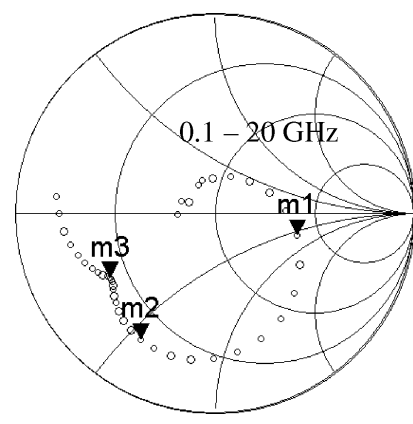

Fig. 3. Smulated loading reflection coefficient for the first-stage transistor of this wide-band LNA. Markers $\mathrm{m} 1, \mathrm{~m} 2$, and $\mathrm{m} 3$ correspond to 5,10 , and $15 \mathrm{GHz}$, respectively. The adjacent circles are $0.5 \mathrm{GHz}$ apart. By treating the 8-20-GHz contour on the lower half of Smith chart as that from an $R C$ circuit, the characteristics of the wide-band LNA can be easily explained.

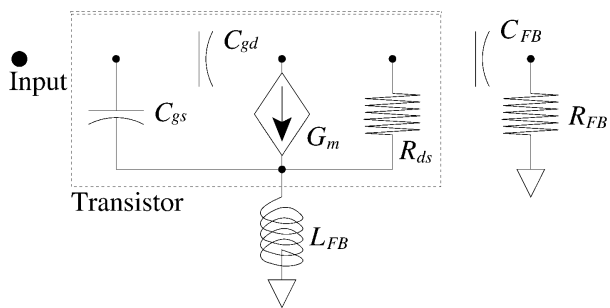

(a)

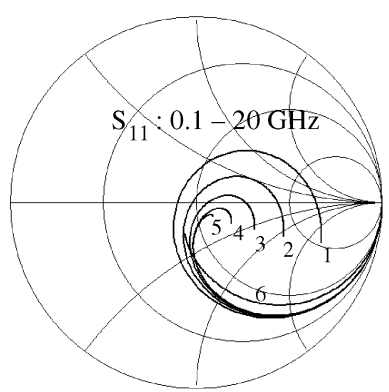

(b)

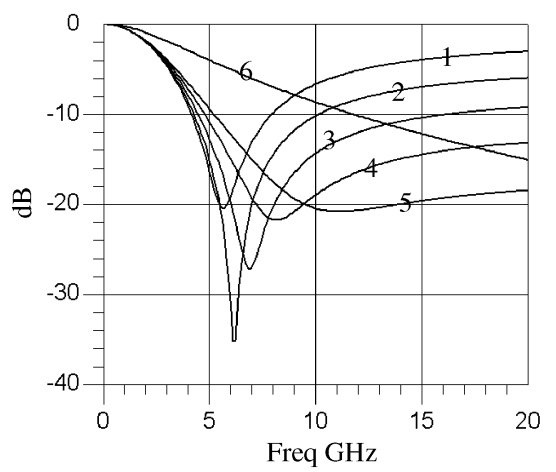

(c)

Fig. 4. Wide-band input match using intrinsic gate-drain capacitor $C_{\text {gd }}$. (a) Schematic. The dotted box is the transistor used in the simulated. The $R C$ loading impedance is coming from the second-stage circuit of the LNA. (b) The $S_{11}$ of this transistor circuit with $C_{\mathrm{gs}}=113 \mathrm{fF}, C_{\mathrm{gd}}=59 \mathrm{fF}, G_{m}=$ $175 \mathrm{mS}, R_{\mathrm{ds}}=50 \Omega, C_{F B}=457 \mathrm{fF}$, and $L_{F B}=200 \mathrm{pH}$. Curves $1-5$ correspond to $R_{F B}=0,10,20,30$, and $40 \Omega$, respectively, while curve six is with $R_{F B}=50 \Omega$ and no $C_{F B}$. Curves $2-5$ can be interpreted as combinations of low-frequency capacitive feedback (curve 1) and high-frequency inductive feedback (curve 6). (c) $S_{11}$ in dB. An appropriate combination of the loading $R C$ impedance does result in a wide-band input match (curve 5).

inclusion of $L_{F B}$ will alter the transistor's original $S_{11}$ into curves each with a different $\operatorname{Re}\left[Z_{\text {in }}\right]$. To eliminate the capacitive 
part of $Z_{\text {in }}$, an additional input series inductor $L_{\text {series }}$ can be added. However, since a complete input match occurs only at one frequency, this inductive feedback is deemed narrow-band. When $L_{\text {series }}$ is small or nonexistent, the matched frequency will be very high.

The capacitive-feedback mechanism, as in Fig. 4(a) but without the $R_{F B}$, results in a low-frequency input match. Neglecting $C_{\mathrm{gs}}$, the input admittance $Y_{\text {in }}$ can be expressed analytically

$$
\begin{aligned}
Y_{\mathrm{in}} & =j \omega C_{\mathrm{gd}}+\frac{G_{m} C_{\mathrm{gd}}}{C_{F B}} \\
& \cdot\left[1+\frac{1}{j \omega C_{F B} R_{\mathrm{ds}}}+j \omega \frac{L_{F B}}{R_{\mathrm{ds}}}\left(1+G_{m} R_{\mathrm{ds}}\right)\right]^{-1} .
\end{aligned}
$$

This is equivalent to a small capacitor $C_{\mathrm{gd}}$ in shunt with a $R L C$ circuit where $R$ is determined by $C_{F B}, C$ is decided by $R_{\mathrm{ds}}$, and $L_{F B}$ is used to remove the $C$ at one specific frequency. For example, if $C_{F B}=457 \mathrm{fF}$ and $L_{F B}=200 \mathrm{pH}$, the calculated matched frequency is $0.051 / \sqrt{L_{F B} C_{F B}}$, or $5.3 \mathrm{GHz}$. In this feedback mechanism, $\operatorname{Re}\left[Y_{\text {in }}\right]$ is determined by $C_{F B}$ and is totally different from what happens in the inductive feedback.

Since the capacitive feedback can be treated, from the input perspective, as a $R L C$ resonator, a series output resistor $R_{F B}$ could then be introduced as a lossy mechanism to attenuate the resonance and therefore broaden the bandwidth [Fig. 4(b) and (c)]. As long as this $R_{F B}$ resistance is coming from the secondstage circuit rather than a physical resistor, its noise impact can be neglected. A more comprehensive interpretation is that, since the capacitive feedback has a matched input impedance at low frequency, while the inductive feedback (without input $L_{\text {series }}$ ) is matched at high frequency, a combination of both circuit configurations should render a wide-band input match. With a $R_{F B} C_{F B}$ output loading and a $L_{F B}$ on the source of the transistor, this circuit resembles a capacitive feedback at low frequency since the impedance of $C_{F B}$ is much larger than that of $R_{F B}$. When the frequency increases, the capacitive feedback will be replaced by the inductive feedback as $R_{F B} C_{F B}$ is now dominated by $R_{F B}$. The noise temperature of this wide-band transistor circuit is

$$
\begin{aligned}
T_{n}= & \frac{T_{\mathrm{drain}}}{G_{m}^{2} R_{\mathrm{ds}} G_{g}} \\
& \times\left|G_{g}+j\left(X_{g}+\frac{\omega C_{\mathrm{gs}}}{1+j \omega L_{F B} \beta G_{m}}+\omega C_{\mathrm{gd}}\right)\right|^{2}
\end{aligned}
$$

where $\beta=R_{\mathrm{ds}} /\left(R_{\mathrm{ds}}+j \omega L_{F B}\right)$. The inclusion of the source inductor $L_{F B}$ can reduce the effective capacitance of $C_{\mathrm{gs}}$, and thus flattens $T_{n}$ as frequency increases. This explains why this wide-band LNA has a low and relatively flat noise temperature.

\section{CONCLUSION}

In this letter, an $8-20-\mathrm{GHz}$ wide-band LNA has been designed and measured. A comprehensive wide-band mechanism has also been proposed to explain the small input reflection coefficient and low noise temperature intended. This LNA can be cooled down in the liquid Helium cryostat to have a $10-\mathrm{K}$ noise temperature and therefore be used as an ultra-sensitive cryogenic amplifier in the radio-telescope receivers [10].

\section{ACKNOWLEDGMENT}

The author wishes to thank Dr. S. Weinreb, M. Egdar, F. Rice, D. Miller, M. Yang, G. Chattopadhyay, Dr. J. Zmuidzinas, California Institute of Technology, Pasadena, Dr. G. Rebeiz, The University of Michigan at Ann Arbor, and Dr. F. K. Y. Lo, National Radio Astronomy Observatory, Charlottesville, VA, for their support and encouragement.

\section{REFERENCES}

[1] F. Rice, M. Summer, J. Zmuidzinas, R. Hu, H. G. Luduc, A. I. Harris, and D. Miller, "SIS mixer design for a broadband millimeter spectrometer suitable for rapid line surveys and redshift determinations," in Proc. SPIE Conf., vol. 4855, Feb. 2003, pp. 301-311.

[2] N. Wadefalk et al., "Cryogenic wideband ultra-low-noise IF amplifiers operating at ultra-low dc power," IEEE Trans. Microwave Theory Tech., vol. 51, pp. 1705-1711, June 2003.

[3] S. Weinreb, D. L. Fenstermacher, and R. W. Harris, "Ultra-low-noise 1.2-1.7-GHz cooled GaAsFET amplifiers," IEEE Trans. Microwave Theory Tech., vol. MTT-82, pp. 849-853, June 1982.

[4] S. Padin and G. G. Ortiz, "A cooled 1-2-GHz balanced HEMP amplifier," IEEE Trans. Microwave Theory Tech., vol. 39, pp. 1239-1243, June 1991.

[5] M. W. Pospieszalski, "On the noise parameters of isolator and receiver with isolator at the input," IEEE Trans. Microwave Theory Tech., vol. MTT-34, pp. 451-453, June 1986.

[6] I. Lopaz-Fernandez et al., "Wideband ultra-low noise cryogenic InP IF amplifiers for the Herschel mission radiometers," in Proc. SPIE Conf., vol. 4855, Feb. 2003, pp. 489-500.

[7] M. Berroth and R. Bosch, "Broad-band determination of the FET smallsignal equivalent circuit," IEEE Trans. Microwave Theory Tech., vol. 38, pp. 891-895, July 1990.

[8] G. Dambrine, A. Cappy, F. Heliodore, and E. Playez, "A new method for determining the FET small-signal equivalent circuit," IEEE Trans. Microwave Theory Tech., vol. MTT-36, pp. 1151-1159, July 1988.

[9] M. W. Pospieszalski, "Modeling of noise parameters of MESFETs and MODFETs and their frequency and temperature dependence," IEEE Trans. Microwave Theory Tech., vol. MTT-37, pp. 1340-1350, Sept. 1989.

[10] R. Hu and S. Weinreb, "A novel wide-band noise-parameter measurement method and its cryogenic application," IEEE Trans. Microwave Theory Tech., vol. 53, pp. 1498-1507, May 2004. 